\title{
¿Es concebible una institucionalización de la educación olímpica?
}

\author{
Éric Monnin* \\ Recibido: 23-11-2020 \\ Aceptado: 24-1 1-2020 \\ Citar como: Monnin, É. (2021). ¿Es concebible una institucionalización de la educación olímpica? \\ Revista Interamericana de Investigación, Educación y Pedagogía, 14(2), 71-112. \\ https://doi.org/10.15332/25005421.6638
}

\section{Resumen}

Mucho antes de que los Juegos de la XXXIII Olimpiada se adjudicaran a la ciudad de París en 2024, el Ministerio de Educación Nacional, Enseñanza Superior e Investigación (MENESR) desplegó varios programas de gran envergadura para apoyar la candidatura mediante el Año del deporte de la escuela a la universidad (2015-2016) o el Año del olimpismo de la escuela a la universidad (2016-2017). Como señala el Ministerio, se trata de vincular la práctica del deporte a una ambición educativa, cultural o cívica basada en el olimpismo y sus valores. Desde la designación de París como ciudad anfitriona de los Juegos de la Olimpiada (13 de septiembre de 2017), el comité organizador ha propuesto la aplicación de herramientas educativas en el sistema escolar y universitario, así como la etiqueta Generación 2024 destinada a fomentar el desarrollo de proyectos de estructuración o la participación en eventos de promoción de las olimpiadas y paralimpiadas. Para una ciudad anfitriona, esta es una oportunidad real de promover y desarrollar la educación a través de los Juegos Olímpicos.

\footnotetext{
* Vicepresidente de la Universidad de Franche-Comté; delegado para el olimpismo - Generación 2024; director del Centro de Estudios e Investigaciones Olímpicas Universitarias (CEROU); profesor asociado (Ph. D. / HDR). Integrante del Laboratorio de Cultura, Deporte, Salud y Sociedad (EA 4660) de la Universidad de Franche-Comté, UPFR Deportes.

Correo electrónico: eric.monnin@univ-fcomte.fr
} 
Palabras clave: olimpismo, Generación 2024, sistema educativo, etiqueta, programas, París 2024.

\title{
Is the Institutionalization of Olympic Education Conceivable?
}

\begin{abstract}
Long before the Games of the XXXIII Olympiad were awarded to the city of Paris in 2024, the Ministry of National Education, Higher Education, and Research (MENESR) deployed different large-scale programs to support the nomination through the Year of Sport from School to University (2015-2016) or the Year of Olympism from School to University (2016-2017). As the Ministry points out, the aim is to link the practice of sport to an educational, cultural, or civic ambition based on Olympism and its values. Since the designation of Paris as the host city for the Games of the Olympiad (September 13, 2017), the organizing committee has proposed the implementation of educational tools in the school and university system, as well as the Generation 2024 label aimed at encouraging the development of structuring projects or the participation in events promoting the Olympics and Paralympics. For a host city, this is a real opportunity to promote and develop education through the Olympic Games.
\end{abstract}

Keywords: Olympism, Generation 2024, education system, label, programs, Paris 2024.

\section{É possível conceber uma institucionalização da educação olímpica?}

\section{Resumo}

Muito antes que a edição XXXIII dos Jogos Olímpicos fosse concedida à cidade de Paris em 2024, o Ministério de Educação 
Nacional, Ensino Superior e Pesquisa (MENESR, na sigla em francês) lançou vários programas de grande peso para apoiar a candidatura por meio do Ano do esporte da escola à universidade (2015-2016) ou o Ano do olimpismo da escola à universidade (2016-2017). Como indica o Ministério, trata-se de vincular a prática do esporte a uma ambição educativa, cultural ou cívica baseada no olimpismo e seus valores. Desde a designação de Paris como cidade-sede dos Jogos Olímpicos (13 de setembro de 2017), o comitê organizador propôs a aplicação de ferramentas educativas no sistema escolar e universitário, bem como o título Geração 2024 destinado a fomentar o desenvolvimento de projetos de estruturação ou a participação em eventos de promoção das Olimpíadas e Paraolimpíadas. Para uma cidade-sede, essa é uma oportunidade real de promover e desenvolver a educação por meio dos Jogos Olímpicos.

Palavras-chave: olimpismo, Geração 2024, sistema educativo, título, programas, Paris 2024.

\section{Introducción}

Al término de los debates del 23 de junio de 1894, los miembros del Congreso Internacional de París para la Restauración de los Juegos Olímpicos ratificaron los informes de las comisiones y anunciaron oficialmente la creación del Comité Olímpico Internacional (COI), presidido por el griego Demetrius Vikelas (1835-1908). Pierre de Coubertin restableció los juegos olímpicos (olimpiadas) de la antigüedad y creó una estructura deportiva internacional.

Desde 1894, el COI ya ha tenido que resolver muchas crisis políticas y sanitarias. Por ejemplo, en 1908 durante los Juegos de la IV Olimpiada de Londres se produjeron dos incidentes políticos: los rusos se opusieron a la presencia de la bandera finlandesa y los británicos a la presencia de la bandera irlandesa. En 2016, durante los Juegos de la XXXI Olimpiada de Río de Janeiro, 
los organizadores brasileños se enfrentaron a una nueva epidemia, el virus Zika.

El 24 de marzo de 2020, el presidente del COI, Thomas Bach, tomó una decisión histórica frente a la pandemia de la COVID-19: el aplazamiento de los Juegos de la XXXII Olimpiada en Tokio. Para combatir los riesgos del sedentarismo y la inactividad relacionados con el confinamiento, en el Día Olímpico (23 de junio de 2020), el COI, la Organización Mundial de la Salud (OMS) y las Naciones Unidas (NN. UU.) están aunando esfuerzos a través del programa \#Healthy Together para fomentar un comportamiento que ayude a las personas a mantenerse física y mentalmente sanas, y a frenar la pandemia. Unas semanas antes, el 16 de mayo, el COI firmó un memorando con la OMS para unirse a su campaña Healthy at Home y beActive, y lanzar la tercera edición de Walk the Talk, From words to action, the health challenge for all.

Antes de la decisión de posponer los Juegos de la XXXII Olimpiada en Tokio, se celebraron los terceros Juegos Olímpicos Juveniles de Invierno (JOJ), que se llevaron a cabo en enero de 2020 en la ciudad suiza de Lausana.

Más allá de las crisis sanitarias, políticas o geopolíticas, en la sociedad actual el olimpismo ocupa un lugar importante a través de las olimpiadas. Para el COI es sobre todo:

[...] una filosofía de vida, exaltando y combinando en un todo equilibrado las cualidades del cuerpo, la voluntad y la mente. Combinando el deporte con la cultura y la educación, el olimpismo trata de crear un estilo de vida basado en la alegría del esfuerzo, el valor educativo del buen ejemplo y el respeto de los principios éticos fundamentales universales. (Comité Olímpico Internacional, 2020a, p. 11)

De acuerdo con la nueva Agenda Olímpica 2020 (Comité Olímpico Internacional, 2014) elaborada y adoptada por los miembros del COI en 2014, una de las prioridades es reforzar, a través de las olimpiadas, 
los Juegos de Invierno (JOJ), los principios fundamentales del olimpismo y la difusión de los valores olímpicos, mediante programas educativos y de formación.

Con este espíritu, el Comité Organizador de la XXXIII Olimpiada de París pretende que estos futuros juegos sean un:

Gran acontecimiento en la historia de los mismos. La generación joven, la Generación 2024, [que] se le ofrecerán programas innovadores integrados en los programas escolares nacionales y que utilizará los juegos como palanca para estimular el espíritu empresarial, el deporte y las oportunidades cívicas y humanitarias. (París, ciudad candidata a los Juegos Olímpicos de 2024, 2016, p. 44)

Las Olimpiadas, las Olimpiadas de Invierno y los JOJ parecen ser una oportunidad para crear una dinámica dentro del sistema educativo en escuelas, colegios, institutos y universidades. A pesar de esta voluntad declarada, ¿la organización de los acontecimientos olímpicos y la aplicación de las herramientas educativas olímpicas pueden facilitar la enseñanza y responder a las cuestiones educativas, deportivas, culturales y sociales actuales? ¿qué tipos de medios e instrumentos se utilizan para desarrollar esta educación olímpica?

Nuestra presentación se centrará, en primer lugar, en los programas institucionales y las herramientas olímpicas disponibles. A continuación, analizaremos las acciones emblemáticas realizadas dentro del sistema educativo como parte del legado de París 2024. Finalmente, concluiremos esta reflexión haciendo referencia a los Centros de Estudios Olímpicos Universitarios (OSC), más precisamente al primer Centro de Estudios e Investigación Olímpica Universitario Francés (CEROU). 


\section{Programas institucionales $e$ instrumentos olímpicos}

\section{Comisión del COI para la Cultura y la Educación Olímpica}

Durante varios años, el COI ha realizado una serie de estudios, encuestas, programas, etc. sobre la educación, la cultura y los Juegos Olímpicos.

Para la preparación de los Juegos de la Olimpiada XXIX de 2008 en Beijing (China), la Comisión de Cultura y Educación Olímpica del COI trabajó en la creación de un Programa de Educación en Valores Olímpicos (OVEP) destinado a alentar a los maestros y entrenadores de todo el mundo para introducir los valores olímpicos en sus programas y actividades educativas (Comité Olímpico Internacional, 2006).

En septiembre de 2008, el OVEP se presentó oficialmente en el Foro Mundial sobre el Deporte, la Educación y la Cultura, celebrado en Busan (Corea del Sur). Este programa tiene tres componentes:

- Un manual educativo Enseñando valores. Un Manual de Educación Olímpica (Comité Olímpico Internacional, 2007), lanzado oficialmente en octubre de 2008.

- Una etiqueta para los usuarios de OVEP a partir de octubre de 2009 (Comité Olímpico Internacional, 2008).

- Una base de datos interactiva disponible oficialmente desde diciembre de 2009.

Según lo expresado por el presidente de la Comisión, Zhenliang Hé:

La unión del deporte, la cultura y la educación está en la base del Movimiento Olímpico y ha sido la fuerza motriz 
del olimpismo durante el último siglo. No cabe duda de que, incluso hoy en día, el deporte sigue teniendo una función educativa esencial en nuestras sociedades a todos los niveles, desde el deporte escolar hasta el deporte de élite y el deporte de masas. El deporte y la educación también han extendido su influencia a muchas otras áreas como la salud, el medio ambiente o la capacidad de gestión. (Comité Olímpico Internacional, 2006)

Sobre la base de este trabajo inicial, en octubre de 2016, el COI desarrolló una versión 2.0 del OVEP. Al comienzo del año escolar 2017, se dispuso de un kit que constaba de cuatro recursos a los cuales se podía acceder de forma gratuita (Comité Olímpico Internacional, 2020b):

- Los fundamentos de la educación en valores olímpicos. Un programa basado en el deporte (manual).

- Implementación del OVEP: una guía práctica para la educación en los valores olímpicos.

- Hojas de trabajo: ejercicios para apoyar la educación en los valores olímpicos.

- Plan del taller sobre el OVEP: aprendizaje a través de la actividad física.

EI PEVO [es una herramienta] puede enriquecer los recursos educativos existentes, contribuir a mejorar el rendimiento académico y fomentar la responsabilidad cívica mediante su enfoque basado en la práctica. El respeto de la diversidad cultural y los sistemas educativos son condiciones esenciales para el programa. (Comité Olímpico Internacional, 2020c) 
Regreso a la escuela y a la universidad 2015-2016: Año del deporte de la escuela a la universidad

Un mes antes de la declaración oficial de la candidatura de París al COI, Najat Vallaud-Belkacem, ministra de Educación Nacional, Enseñanza Superior e Investigación, anunció el 18 de mayo de 2015, en apoyo de la candidatura de París 2024, la operación Año del deporte de la escuela a la universidad para el año 2015-2016. El objetivo principal era vincular la práctica del deporte a una ambición educativa, cultural o cívica, y promover así el deporte dentro del sistema educativo francés, apoyándose en los grandes campeonatos europeos (baloncesto, campo a través, bádminton y fútbol) organizados en Francia (Ministerio de Educación Nacional, Enseñanza Superior e Investigación, 2015).

Para facilitar la puesta en marcha de las acciones, se creó una plataforma dedicada a esta operación, la cual permitía enumerar el conjunto de los recursos pedagógicos puestos a disposición por la red Canopée y accesibles en la plataforma Éduscol. Además del Ministerio, que dirigía el proyecto, se establecieron diversas asociaciones a nivel nacional, con variaciones regionales, con los Ministerios de Asuntos Urbanos y de Juventud y Deportes, las federaciones deportivas escolares y universitarias, así como las federaciones deportivas civiles encargadas de los campeonatos europeos mencionados, y el Comité Nacional Olímpico y Deportivo de Francia (CNOSF). Con esto, se buscaba alcanzar los siguientes objetivos:

- Promover las prácticas deportivas desde la escuela hasta la universidad. En primer lugar, la educación física y deportiva (PES) y el deporte escolar.

- El deporte es una herramienta pedagógica que contribuye a las diferentes áreas de formación en la educación.

- El deporte es un instrumento que refuerza los vínculos entre las instituciones educativas, su entorno y el 
medio asociativo. (Ministerio de Educación Nacional, Enseñanza Superior e Investigación, 2015)

En total, 1961 proyectos fueron etiquetados como Año del deporte desde la escuela a la universidad, el $40 \%$ de ellos en la escuela primaria, principalmente a través de actividades multideportivas centradas en dos temas: la cultura del deporte y la educación para la salud. Entre todos estos proyectos, "un jurado nacional seleccionó 35 ganadores de entre las 148 solicitudes escogidas, para otorgar diez premios de 5000 EUR y 25 premios de 2000 EUR a los jefes de proyecto" (Frécon, 2016, pp. 38-39).

Para la ministra Vallaud-Belkacem, esta promoción del deporte en la escuela y la universidad se ajustó plenamente a la Ley n. ${ }^{\circ}$ 2013-595 del 8 de julio de 2013, relacionada con la orientación y la programación de la refundación de la Escuela de la República, así como al decreto n. ${ }^{\circ} 2015-372$ del 31 de marzo de 2015, donde se describe la base común de conocimientos, competencias y cultura, organizada en tres ciclos de enseñanza, que determinan los conocimientos y competencias esenciales que deben adquirirse al final de la escolaridad obligatoria.

A través del Año del deporte desde la escuela a la universidad se movilizan las cinco áreas del núcleo común de conocimientos, habilidades y cultura, impulsando la Educación Moral y Cívica (EMC) mediante "la promoción de la práctica deportiva entre los jóvenes y la movilización de toda la comunidad educativa en torno a los valores cívicos y deportivos" (Frécon, 2016, p. 39).

Para mantener este impulso, el MENESR decidió, en su circular n. ${ }^{\circ} 30$ del 25 de agosto de 2016 y siempre en apoyo de la candidatura de París 2024, hacer del año escolar 2016-2017, el año del olimpismo, para:

Promover la práctica del deporte entre los jóvenes y movilizar a la comunidad educativa en torno a los valores cívicos y deportivos en el marco de un Año del Olimpismo, de la Escuela a la Universidad y realizar acciones educativas 
en este sentido a lo largo de todo el año. (Ministerio de Educación Nacional, Enseñanza Superior e Investigación, 2016)

De forma simbólica, el Año del deporte de la escuela a la universidad terminó el miércoles 14 de septiembre de 2016, misma fecha en la que se celebra el Día Nacional del Deporte Escolar (JNSS), con la inauguración de la operación Año del Olimpismo de la Escuela a la Universidad (Ministerio de Educación Nacional, 2016).

Regreso a la escuela y a la universidad 2016-2017: Año del olimpismo de la escuela a la universidad

Para el MENESR, la candidatura de París 2024 es una verdadera oportunidad proyectar sus sistemas existentes, como el camino de la ciudadanía del estudiante, el camino de la educación sanitaria o el camino de la educación moral y cívica (EMC) creados mediante la Ley n. ${ }^{\circ}$ 2013-595 del 8 de julio de 2013 sobre la orientación y la programación de la refundación de la École de la République y aplicados desde el inicio del año escolar 2015.

Durante esta nueva operación, se dio prioridad a cuatro objetivos principales:

- Valorizar las actividades deportivas en la escuela, en primer lugar la educación física y el deporte (PES), y el deporte escolar y universitario.

- Promover el deporte como instrumento pedagógico para contribuir a las diferentes áreas de formación en la educación escolar y universitaria.

- Dar dimensión cultural al deporte, mediante el desarrollo de actividades tanto culturales como deportivas.

- Movilizar el deporte como herramienta para reforzar los vínculos entre las instituciones educativas, su entorno 
y el movimiento deportivo, y así corregir las desigualdades en el acceso al deporte cualesquiera que sean las causas: territoriales, sociales, de género, culturales o relacionadas con la discapacidad

(Ministerio de Educación Nacional, 2016).

Para cumplir con estos objetivos, el MENESR renovó su tradicional colaboración con el Ministerio de Juventud y Deportes, la escuela, la universidad (Unión deportiva de la enseñanza de primer grado, Unión nacional del deporte escolar, Unión general deportiva de la enseñanza libre, Federación francesa del deporte universitario) y las federaciones deportivas civiles, la Comité Olímpico y Deportivo Nacional de Francia y el Comité Paralímpico y Deportivo Francés (CPSF). Estas asociaciones fueron transmitidas a nivel regional y local por el Movimiento Deportivo (Comité Olímpico y Deportivo Regional, Comité Olímpico y Deportivo Departamental, comités regionales y departamentales, clubes y asociaciones) y por las comunidades educativas (asociaciones de padres, asociaciones de estudiantes, etc.) presentes en cada academia.

Como continuidad del Año del deporte desde la escuela a la universidad, se renovaron en su totalidad los comités de direcciones nacionales y regionales; las convocatorias de proyectos; la etiqueta; la aportación de recursos a través de la red Canopée; y el portal del Ministerio de Educación Nacional, Éduscol. Una novedad fue el nombramiento de un referente olímpico en ciertas academias.

Como lo recuerda el MENESR, las acciones que se etiquetan deben "vincular la práctica del deporte a una ambición educativa, cultural o cívica en torno al olimpismo y sus valores" (Ministerio de Educación Nacional, 2016).

Cinco grandes eventos marcaron este año olímpico:

- Día Internacional del Deporte Universitario, 20 de septiembre de 2016.

- Día Nacional de los Deportes Escolares (NSSD), 14 de septiembre de 2016. 
- Día del Deporte en el Campus, a principios de octubre de 2016.

- Semana Olímpica y Paralímpica, 21-29 de enero de 2017 (1. ${ }^{\text {a }}$ edición).

- Día Olímpico, 23 de junio de 2017.

Gracias a esta operación y a los 700 proyectos etiquetados, 98 de los cuales recibieron apoyo financiero del MENESR, más de 2 millones de alumnos fueron sensibilizados sobre el olimpismo y sus valores, mediante proyectos relacionados con la cultura, el deporte, la salud, la ciudadanía, el medio ambiente, la convivencia en sociedad, etc.

\section{En 2017, programa interministerial: \\ El deporte al servicio de la sociedad}

Unos meses antes del anuncio del COI de designar la ciudad sede de los Juegos Olímpicos de 2024, el ministro de la Ciudad, la Juventud y el Deporte y el secretario de Estado para el Deporte presentaron ante el Consejo de Ministros, el 22 de marzo de 2017, un programa interministerial, con el fin de garantizar el apoyo del Estado y especificar el legado olímpico que dejará la organización de los Juegos Olímpicos y Paralímpicos de 2024. Se liberó un presupuesto de 20000000 EUR para un plan de desarrollo deportivo.

Las actas del Consejo de Ministros establecen claramente que:

El legado es un elemento importante de la candidatura de París 2024, que desea responder plenamente a las expectativas de los franceses proponiendo un proyecto sostenible al servicio de la sociedad. Por ello, el Estado aplica actualmente un programa de apoyo interministerial en el marco de una dinámica de legado llevada a cabo por el Grupo de Interés Público (GIP) París 2024. (Consejo de Ministros, 2017) 
En un documento de apoyo titulado El deporte al servicio de la sociedad (París, ciudad candidata a los Juegos Olímpicos de 2024 y Ministerio de la Ciudad, la Juventud y los Deportes, 2017) se enumeran las 24 medidas de este programa interministerial desarrolladas a través de seis temas: juventud/educación/cultura, salud/discapacidad, integración social/empleo, igualdad de género, deporte y territorios, excelencia medioambiental.

Entre las cinco medidas del tema de la juventud/educación/cultura, la primera se refiere a la creación de la etiqueta Generación 2024 para escuelas y universidades. El objetivo es "[...] desarrollar la práctica del deporte entre los jóvenes mediante la aplicación de acciones concretas y multiplicadoras a nivel nacional" (París, ciudad candidata a los Juegos Olímpicos de 2024 y Ministerio de la Ciudad, la Juventud y los Deportes, 2018, p. 4).

Durante este año escolar, se programaron tres momentos destacados:

- Día Nacional de los Deportes Escolares

- Semana Olímpica y Paralímpica

- Día Olímpico.

Más allá de los criterios para obtener la etiqueta (participación en la Semana Olímpica y Paralímpica), los objetivos se muestran claramente a través de los siguientes indicadores:

- Etiquetar al menos el $20 \%$ de los establecimientos para 2024.

- Promover la apertura, fuera del horario escolar, de 16.000 instalaciones deportivas escolares para el 2024.

- Involucrar a las 63.000 escuelas, colegios e institutos franceses en la semana olímpica. 
- Elaborar y difundir una guía de buenas prácticas: Cómo acoger a un deportista de alto nivel en su escuela.

- Organizar la firma de una carta, con el fin de promover el deporte y el PSE como herramientas para la igualdad entre niñas y niños con los ministerios correspondientes y las federaciones deportivas escolares y universitarias.

Con ocasión de la octava Jornada Nacional del Deporte Escolar, que se celebró el miércoles 27 de septiembre del año escolar 2017-2018, el Ministro de Educación Nacional visitó el complejo deportivo Jules Ladoumègue de París. Asimismo, pocos días después de la designación de París como sede de los Juegos Olímpicos y Paralímpicos de 2024, asumió siete compromisos, entre ellos el de:

Una convocatoria de proyectos de "Etiquetado de la generación 2024" [que] se lanzará en colaboración con el Ministerio de Deportes para las escuelas y los establecimientos educativos, para su aplicación desde el inicio del año escolar 2018, con el objetivo de un centenar de etiquetas en el primer año. (Ministerio de Educación Nacional, Juventud y Deportes, 2020a)

Las especificaciones de la etiqueta Generación 2024 incluyeron cuatro objetivos (Ministerio de Educación Nacional y Juventud, Ministerio de Agricultura y Alimentación y Ministerio de Deportes, 2018):

- Desarrollar proyectos de estructuración con los clubes deportivos locales.

- Participar en los eventos promocionales de los Juegos Olímpicos y Paralímpicos.

- Adaptar los cursos de los deportistas de alto nivel.

- Abrirlasinstalaciones deportivas de los establecimientos. 
A estos objetivos, se añadieron cuatro principios rectores:

- Fomentar el trabajo voluntario en escuelas e instituciones, para desarrollar la práctica del deporte.

- Fomentar la autonomía de las escuelas/instituciones, el acceso a nuevos métodos de asociación educativa.

- Fomentar la continuidad educativa en las diferentes etapas de la vida de un joven, en colaboración con las autoridades locales y el mundo deportivo local.

- Depender de la experiencia de la asociación deportiva de la escuela o EPLE (USEP, UNSS, UGSEL).

Sin esperar el comienzo del nuevo año escolar, 269 escuelas y establecimientos educativos (128 escuelas primarias, 67 escuelas medias, 70 escuelas secundarias y 4 establecimientos de formación agrícola) recibieron la etiqueta Generación 2024. Para el comienzo del año escolar 2018, se proyectaba que un total de 985 escuelas y establecimientos (411 escuelas, 378 colegios y 196 liceos) hubiesen recibido esta etiqueta. En la actualidad, se han etiquetado 2212 escuelas, colegios e institutos (Ministerio de Educación Nacional y Juventud, 2019), así como 65 universidades, grandes escuelas y el Centro de Recursos y Experiencias para el Rendimiento Deportivo (tabla 1) (Ministerio de Educación Superior, Investigación e Innovación, 2020).

Tabla 1. Número de instituciones de educación superior etiquetadas como Generación 2024 en 2020.

\begin{tabular}{|l|c|c|c|c|}
\hline & $\begin{array}{c}4 \text { de febrero de } \\
\mathbf{2 0 1 9}\end{array}$ & $\begin{array}{c}22 \text { de junio de } \\
\mathbf{2 0 1 9}\end{array}$ & $\begin{array}{c}23 \text { de junio de } \\
\mathbf{2 0 2 0}\end{array}$ & Total \\
\hline Universidades & 24 & 15 & 3 & 42 \\
\hline $\begin{array}{l}\text { Grandes } \\
\text { escuelas }\end{array}$ & 6 & 4 & 4 & 14 \\
\hline CREPS & & 6 & 3 & 9 \\
\hline Total & 30 & 25 & 10 & 65 \\
\hline
\end{tabular}

Fuente: elaboración propia. 


\section{Acciones emblemáticas realizadas dentro del sistema educativo como parte del legado de París 2024}

Como se mencionó, el documento interministerial El deporte al servicio de la sociedad en 2017 enumera las 24 medidas necesarias para dejar un legado duradero de París 2024 (París como ciudad candidata a los Juegos Olímpicos de 2024 y el Ministerio de la Ciudad, la Juventud y los Deportes, 2017). La primera medida se refiere a la creación de la etiqueta Generación 2024 para escuelas y universidades. También es una oportunidad para insistir en los tres puntos altos programados durante el año escolar y universitario:

- Día Nacional de los Deportes Escolares, en septiembre.

- Semana Olímpica y Paralímpica, en febrero.

- Día Olímpico, en junio (tabla 2).

Tabla 2. Principales acontecimientos del año escolar y universitario 2016-2017.

\begin{tabular}{|l|c|}
\hline Evento & 1. ${ }^{\text {a edición }}$ \\
\hline Día Nacional de los Deportes Escolares & 2010 \\
\hline Semana Olímpica y Paralímpica & 2017 \\
\hline Día Olímpico & 1948 \\
\hline
\end{tabular}

Fuente: elaboración propia.

\section{Día Nacional de los Deportes Escolares (NSSD)}

El 25 de mayo de 2010, el Ministro de Educación Nacional anunció la creación del Día del Deporte Escolar, con una primera reunión que se llevó a cabo el día miércoles 22 de septiembre de 2010. El objetivo era iniciar el año escolar y reanudar las actividades físicas en todas las escuelas.

La 11. a edición de la JNSS sobre el tema la salud y la reanudación de la actividad, se celebró el día miércoles 23 de septiembre de 2020, 
por iniciativa del Ministerio de Educación Nacional, Juventud y Deportes. El objetivo de este día es "sensibilizar y promover las actividades propuestas por las asociaciones y federaciones deportivas escolares (UNSS, USEP y UGSEL) entre los estudiantes, los equipos educativos, los padres, el mundo deportivo local y las autoridades locales" (Ministerio de Educación Nacional, Juventud y Deportes, 2020b).

También es una oportunidad para promover los valores del deporte y el olimpismo en la escuela.

El Día Nacional de los Deportes Escolares es una oportunidad para concienciar sobre los valores del olimpismo y el paralelismo, utilizar el deporte como herramienta educativa, introducir a los estudiantes en las disciplinas olímpicas y paralímpicas, cambiar la visión de los jóvenes sobre la discapacidad y despertar en ellos el compromiso voluntario. La noción de legado es una de las principales cuestiones en juego en la organización de los Juegos Olímpicos y Paralímpicos de París, donde el Día Nacional del Deporte Escolar desempeña un papel fundamental en la construcción de este legado. (Navarranne, 2019)

Esta JNSS es parte de la Semana Europea del Deporte iniciada en 2015 por la Comisión Europea. En 2019, 42 países europeos participaron en la Semana Europea del Deporte, que reunió a más de 15 millones de participantes en los 28300 proyectos propuestos.

\section{Semana Olímpica y Paralímpica (SOP)}

La SOP se sitúa entre el Día Nacional del Deporte Escolar (1. ${ }^{a}$ edición en 2010) programado en septiembre y el Día Olímpico del 23 de junio instituido por el COI en 1948. Asimismo, complementa las clases olímpicas, un sistema lanzado hace varios años por el Comité Nacional Olímpico y Deportivo Francés (CNOSF).

El Comité Organizador de París 2024 decidió inaugurar la fase de candidatura de la SOP en 2017. Esta candidatura estuvo abierta 
a todas las escuelas, establecimientos educativos e instituciones de enseñanza superior y para todas las edades, desde el jardín de infancia hasta la universidad.

En febrero de 2020, el tema de la $4 .^{\text {a }}$ edición fue la interculturalidad en relación con los JOJ de Lausana, celebrados en enero de 2020, y los Juegos de la XXXII Olimpiada de Tokio (originalmente previstos para julio de 2020 y aplazados para el 23 de julio de 2021 debido a la pandemia de la COVID-19).

Tras la alarmante constatación en Francia de que el $92 \%$ de las niñas y el $82 \%$ de los niños de 6 a 11 años no se mueven lo suficiente (París, 2024, 2020a), el Ministerio de Educación Nacional, Juventud y Deportes, París 2024, y el movimiento deportivo decidieron, al mismo tiempo, poner en marcha el proyecto de actividad física diaria 30, el cual estuvo en consonancia con la política educativa de la Escuela promotora de la salud (Ministerio de Educación Nacional y Juventud, 2018) iniciada por el primer ministro y el ministro de Educación Nacional y Juventud en el Comité Interministerial de Salud del 26 de marzo de 2018.

A este objetivo de combatir el sedentarismo y promover la actividad física, se sumó una grave crisis de salud, desde que el Director General de la OMS, Tedros Adhanom Ghebreyesus, anunciara el 11 de marzo de 2020 la pandemia causada por la COVID-19. Más que nunca, la salud fue el centro de las preocupaciones de nuestras sociedades. Con el fin de reforzar las medidas aplicadas, el Comité Organizador de París 2024 decidió que, en febrero de 2021, el tema de la 5. ${ }^{a}$ edición de la SOP sería la salud (tabla 3 ).

Tabla 3. Evaluación de las Semanas Olímpicas y Paralímpicas (SOP) de las ediciones de 2018, 2019 y 2020.

\begin{tabular}{|l|c|c|c|c|c|}
\hline & SOP 2017 & SOP 2018 & SOP 2019 & SOP 2020 & SOP 2021 \\
\hline Edición & 1 & 2 & 3 & 4 & 5 \\
\hline $\begin{array}{l}\text { Las instituciones } \\
\text { movilizadas }\end{array}$ & 11000 & 80000 & 180000 & 450000 & - \\
\hline $\begin{array}{l}\text { Estudiantes } \\
\text { sensibilizados }\end{array}$ & - & 100 & 150 & 200 & - \\
\hline $\begin{array}{l}\text { Atletas } \\
\text { movilizados }\end{array}$ & & 500 & 1500 & 2800 & - \\
\hline
\end{tabular}




\begin{tabular}{|l|c|c|c|c|c|}
\hline & SOP 2017 & SOP 2018 & SOP 2019 & SOP 2020 & SOP 2021 \\
\hline Tema del sop & - & - & Mixto & Interculturalidad & Salud \\
\hline $\begin{array}{l}\text { Proyectos etiquetados } \\
\text { presentados }\end{array}$ & 40 & 500 & 1300 & 1600 & - \\
\hline Fechas & $\begin{array}{c}\text { Del 21 al 29 } \\
\text { de enero }\end{array}$ & $\begin{array}{c}\text { Del 27 de } \\
\text { enero al 3 } \\
\text { de febrero }\end{array}$ & $\begin{array}{c}\text { Del 4 al 9 } \\
\text { de febrero }\end{array}$ & $\begin{array}{c}\text { Del 3 al } 8 \text { de } \\
\text { febrero }\end{array}$ & $\begin{array}{c}\text { Del } 1 \text { al } 6 \\
\text { de febrero }\end{array}$ \\
\hline
\end{tabular}

Fuente: elaboración propia.

Como lo menciona el Comité Organizador de París 2024:

La Semana Olímpica y Paralímpica tiene los siguientes objetivos educativos:

- Utilizar el deporte como herramienta pedagógica en la enseñanza.

- Concienciar sobre los valores olímpicos y paralímpicos, movilizando las herramientas educativas y lúdicas disponibles.

- Descubrir las disciplinas olímpicas y paralímpicas en colaboración con el movimiento deportivo, mediante la organización de talleres para la práctica del deporte.

- Cambiar la forma en que vemos la discapacidad, descubriendo los paradeportes e integrando las reuniones paradeportivas o los deportes compartidos.

Despertar a los jóvenes al compromiso voluntario y cívico. La SOP Se organiza en toda Francia, en Francia metropolitana y en ultramar, así como en las escuelas francesas en el extranjero (AEFE). (París 2024, 2020b, p. 4)

Para facilitar la organización de las SOP en las escuelas, el Comité Organizador de París 2024 puso a disposición una serie de recursos educativos: guía SOP 2021, fichas de información para los profesores, carteles de los Juegos Olímpicos, pedagogía en movimiento, los Antiguos Juegos Olímpicos, voleibol sentado (plan de clases para los profesores), ¿cómo acoger a un atleta?, la llama 
olímpica y el relevo (Berlín 1936), secuencia de juego limpio del PES, boletín de la escuela secundaria, los Juegos Paralímpicos (plan de lecciones del profesor), película El color de la victoria.

Todos estos recursos permitirán trabajar con los estudiantes sobre los valores olímpicos y paralímpicos, la historia de los Juegos, el deporte, la discapacidad, los atletas, los símbolos, los medios de comunicación, entre otros aspectos. Además, se dispondrá de los recursos de la CNOSF, como los dosieres pedagógicos para los profesores de los ciclos 1 a 4, desde el jardín de infancia hasta la escuela secundaria.

\section{Día Olímpico}

Al término de los debates del 23 de junio de 1894, los miembros del Congreso Internacional de París para la Restauración de los Juegos Olímpicos, presidido por el Barón de Courcel, ratificaron los informes de las comisiones y anunciaron oficialmente la creación del COI. No fue hasta junio de 1947 (entre el 17 y el 24 de junio) y gracias a Josef Gruss, que se propuso a los miembros del COI, en la 41. ${ }^{a}$ reunión de Estocolmo, un evento para conmemorar la fundación del Movimiento Olímpico y promover los ideales olímpicos en cada país.

En la 42. ${ }^{\text {a }}$ sesión del $\mathrm{COI}$, celebrada en St. Moritz en enero de 1948, los miembros del Comité adoptaron el proyecto de un Día Olímpico Mundial. El primer Día Olímpico fue celebrado el 23 de junio de 1948 por nueve Comité Olímpico Nacional: Austria, Bélgica, Canadá, Portugal, Gran Bretaña, Grecia, Suiza, Uruguay y Venezuela. En 1978, la Carta Olímpica recomendaba que "Ios CON deberían organizar regularmente, si es posible cada año, un Día Olímpico para promover el Movimiento Olímpico" (Comité Olímpico Internacional, 1978, p. 47).

En 1987, con el fin de desarrollar y promover la práctica del deporte, la Comisión Deporte para todos del COI propuso celebrar esta fecha con una carrera del Día Olímpico. 
Hoy en día, el Día Olímpico es mucho más que una carrera o un evento deportivo. Se ha convertido en el único evento anual y mundial que celebra el Movimiento Olímpico. También se ha convertido en un día para la promoción de una vida sana y activa. La organización del Día Olímpico se basa en tres pilares: moverse, aprender y descubrir.

(Comité Olímpico Internacional, 2017 p. 1)

Para la edición de 2020, hubo muchas restricciones debido a la pandemia causada por la COVID-19. Las grandes reuniones se transformaron en carreras virtuales. En Francia:

La CNOSF ha desarrollado un proyecto "híbrido" de Día Olímpico, que combina actividades digitales y físicas, dirigido al público en general, a los jóvenes y a los escolares. Esta acción tiene lugar durante toda la semana del 22 al 26 de junio de 2020, incluyendo el tradicional Día Olímpico el 23 de junio [...] En cuanto al Día Nacional del Deporte Escolar en septiembre y la Semana Olímpica y Paralímpica en febrero, las acciones propuestas deben permitir la participación de todos los estudiantes $y$, por lo tanto, la inclusión más amplia posible. (Ministerio de Educación Nacional, Juventud y Deportes, 2020c).

Para el Día Olímpico, llevado a cabo el 23 de junio de 2020, se propusieron cuatro acciones:

- Las clases se movilizan para el Día Olímpico 2020.

- Corramos juntos para crear el mural del Día Olímpico 2020.

- Trabajando en los valores olímpicos.

- La participación en los desafíos atléticos establecidos por los atletas. 


\section{La etiqueta Generación 2024}

Con el mismo espíritu de la SOP, en 2018 se creó la etiqueta Generación 2024 para las escuelas y universidades. Para las autoridades locales (regiones, departamentos, comunidades de aglomeración, comunidades de municipios, municipalidades) se lanzó en junio de 2019 otra etiqueta Terre de Jeux, con el fin de promover sus territorios. Entre los municipios e intermunicipalidades a los que se les concedió esta última etiqueta, 416 solicitaron el 20 de noviembre de 2019 convertirse en Centro de Preparación para los Juegos y acoger a delegaciones extranjeras en sus infraestructuras.

Para el Ministerio, "[...] el deporte y la escuela se basan en los mismos valores: el gusto por el esfuerzo, la perseverancia, el deseo de progresar, el respeto a los demás, a uno mismo y a las reglas, etc." (Ministerio de Educación Nacional, Enseñanza Superior e Investigación, 2015). La candidatura de París es también un medio de promover las acciones educativas existentes a través de múltiples mecanismos, como el camino de la ciudadanía del estudiante (Ministerio de Educación Nacional y Juventud, 2016). Este trabajo conjunto propone un programa de educación olímpica con oportunidades para estudiantes y deportistas:

[Comprender] los principios fundamentales de la filosofía olímpica [definidos en la Carta Olímpica]; [Presentar] el olimpismo y la idea de los Juegos Olímpicos; [Promover y difundir los valores éticos y educativos del Movimiento Olímpico; para explicar el olimpismo y el ideal universal en el sentido de "Kalokagathia"; para entrenar armoniosamente el cuerpo y la mente de acuerdo con la filosofía olímpica; y para forjar la personalidad de cada individuo de acuerdo con Coubertin, sentando así las bases del entendimiento internacional. (Remmelkoor, 2007, p. 231)

A este respecto, como sugiere Konstantinos Georgiadis, un proyecto de educación olímpica incluye tres puntos principales: 1) comprender los principios olímpicos de la Carta Olímpica; 
2) comprender los fundamentos históricos y pedagógicos del ideal olímpico; 3) comprender mejor nuestra sociedad y los estilos de vida actuales (Georgiadis, 2007).

Con el fin de destacar todas las acciones llevadas a cabo durante este año escolar, el Ministerio de Educación Nacional y de Enseñanza Superior e Investigación otorgó el sello Año del Olimpismo, de la Escuela a la Universidad a 98 proyectos (Revista $E P \& S, 2017)$, que tenían, entre otros, los siguientes objetivos:

- Crear una fuerte asociación entre un establecimiento escolar o de enseñanza superior, un deportista escolar o universitario (federaciones deportivas escolares o universitarias, asociaciones deportivas universitarias) y/o un jugador del movimiento deportivo (CROS, CDOS, federación deportiva, liga, comité o club).

- Vincular la práctica del deporte a una ambición educativa, cultural o cívica en torno al olimpismo y sus valores, basándose en el modelo, por ejemplo, de las "Clases Olímpicas", una acción educativa propuesta por la CNOSF [...]. (Ministerio de Educación Nacional, Enseñanza Superior e Investigación, 2016)

\section{La universidad etiquetó a la Generación 2024}

El 20 de septiembre de 2018, en el Día Internacional del Deporte Universitario (JISU), los ministros de Educación Superior, Investigación e Innovación y Deportes anunciaron oficialmente el lanzamiento de la etiqueta Generación 2024 para las instituciones de educación superior (Monnin, 2019).

Con miras a la organización de los Juegos Olímpicos y Paralímpicos de París en 2024 (JOP 2024), esta etiqueta tiene por objeto fomentar el desarrollo de la práctica regular de los deportes por parte de los estudiantes, en 
particular los becarios y los discapacitados, así como la participación de los estudiantes en el movimiento París 2024. (Ministerio de Enseñanza Superior, Investigación e Innovación, 2019)

De forma paralela a este anuncio, los presidentes de las universidades recibieron dos cartas oficiales de los ministerios correspondientes, en las que se especificaban las expectativas y los objetivos de este etiquetado. La primera carta establecía el marco, retomando todas las cuestiones de sociedad relacionadas con la celebración de los Juegos Olímpicos en Francia: "Nuestra ambición, como saben, es inculcar una verdadera cultura del deporte en nuestro país. Quién mejor que la generación más joven para transmitir estos mensajes" (Carta de los Ministros de Educación Superior, Investigación e Innovación y Deporte, 2018). La segunda carta era técnica, en la cual se indicaba todo el procedimiento y el calendario para presentar una oferta (Plateau, 2018).

Se recordó que esta solicitud es anual y que cada solicitud debía enviarse antes del $1 .^{\circ}$ de diciembre de cada año para ser examinada primero por un consejo técnico a nivel académico (referentes de la Generación 2024 del rectorado y de la dirección regional de Juventud y Deportes y Cohesión Social) y luego por un Comité Nacional de Etiquetado en el mes de enero. Los resultados se anunciarían antes o durante la SOP, que generalmente se celebra en febrero. La etiqueta se concede por un período de tres años.

Para las instituciones de enseñanza superior, esta etiqueta tiene varios objetivos:

- Desarrollar vínculos entre el establecimiento y su entorno de asociación deportiva.

- Participar en los eventos de movilización en torno a los Juegos de París de 2024.

- Abrir las instalaciones deportivas de los establecimientos. 
- Fomentar la práctica regular entre los estudiantes.

- Asegurar que los estudiantes con discapacidades sean tenidos en cuenta.

- Renovar, generalizar y modernizar las condiciones de estudio de los deportistas de alto nivel.

Si se desea obtener la etiqueta Generación 2024, se deben cumplir cuatro puntos (Ministerio de Educación Superior, Investigación e Innovación y Ministerio de Deportes, 2018):

- Desarrollo de vínculos entre el establecimiento y su entorno de asociación deportiva.

- Participación en eventos de investigación y promoción de los Juegos Olímpicos y Paralímpicos.

- Apoyar o acoger a atletas de alto nivel (incluidos los jóvenes con discapacidades).

- Poner a disposición de las asociaciones deportivas y empresas locales las instalaciones deportivas existentes en el campus universitario.

El 4 de febrero de 2019, con motivo del lanzamiento de la SOP, el Ministerio de Enseñanza Superior, Investigación e Innovación y el Ministerio de Deportes concedieron la etiqueta Generación 2024 a la Universidad de Franche-Comté (Universidad de Franche-Comté, 2018a). Es una de las primeras universidades en recibir esta etiqueta, concedida durante tres años. Es un gran reconocimiento para la UFC, que lleva más de diez años aplicando una política comprometida con la promoción del deporte y los valores olímpicos.

Esta etiqueta destaca las diversas acciones llevadas a cabo dentro de la universidad. Por eso, este establecimiento educativo:

- Se distingue de las demás universidades francesas al obtener la primera etiqueta para las instituciones de enseñanza superior. 
- Garantiza una buena visibilidad nacional e internacional en el ámbito del olimpismo, en particular mediante intercambios con universidades extranjeras (profesores investigadores y estudiantes).

- Mantiene la lealtad de sus partidarios, como el COl; el Centro de Estudios Olímpicos (OSC) con sede en Lausana, Suiza; el Comité Nacional Olímpico y Deportivo Francés (CNOSF); etc., para todas las acciones realizadas o por realizar.

- Muestra una fuerte ambición de innovar, apoyándose en los tres eventos promocionales más destacados de los Juegos Olímpicos y Paralímpicos: Día Internacional del Deporte Universitario, Semana Olímpica y Paralímpica, y Día Olímpico.

- Se convierte en la referencia del olimpismo (investigación, publicación) en Francia.

- Se promueve a través de todas sus acciones y conocimientos técnicos dirigidos a los estudiantes (becarios, personas discapacitadas, etc.) y a todo su personal, mediante la organización de ambiciosos eventos: 50 aniversario de los Juegos Olímpicos de Grenoble 2018 en el Palacio de Deportes de Besançon; Día de la Cultura, el Deporte y la Educación; etc.).

- Organiza una serie de actividades deportivas, culturales y artísticas para todos los estudiantes y el personal, aprovechando y promoviendo los numerosos puentes existentes entre la Universidad de Franche-Comté y los clubes deportivos locales, nacionales o internacionales. Todas estas acciones se realizarán en el marco de la estrategia de desarrollo internacional, llevada a cabo por el Departamento de Relaciones Internacionales y de la Francofonía de la Universidad. 


\section{Centros de Estudios Olímpicos (CEO)}

\section{El Comité Olímpico Internacional y los CEO}

Para evitar la confusión con las Academias Olímpicas Nacionales (NOC) y la Academia Olímpica Internacional (IOA) con sede en Olimpia, Grecia, que no son universidades, el COI brinda una definición precisa de lo que es un CEO:

Un Centro Universitario de Estudios e Investigaciones Olímpicas es un centro universitario que funciona gracias a la contribución de profesores, investigadores y estudiantes (de posgrado) y cuya misión es crear o difundir los conocimientos olímpicos, principalmente entre los estudiantes (también se puede dirigir a otros grupos) mediante actividades académicas (cursos, investigaciones, publicaciones y conferencias). Las cátedras olímpicas que corresponden a esta definición también se consideran centros universitarios de estudios e investigación olímpica. (Comité Olímpico Internacional, 2020d, p. 4)

Para beneficiarse del estatus de CEO otorgado por el COI, se deben cumplir cuatro condiciones:

- Ajustarse a la definición de centro universitario de estudios e investigación olímpica.

- Recibir la aprobación de su NOC (carta oficial firmada por el presidente o el secretario general).

- Contar con el apoyo financiero y logístico de la universidad o facultad que acoja al CEO para la realización de un programa anual de actividades (carta oficial firmada por las autoridades universitarias competentes). 
- Para una iniciativa en una ciudad anfitriona destinada a contribuir al legado de los Juegos Olímpicos, recibir una confirmación por escrito del Comité Organizador de los Juegos Olímpicos (COJO) de que la iniciativa está en consonancia con los planes de legado y que se ha establecido una clara coordinación con el COJO, el CON y otras partes que participan en la gestión del legado. (Comité Olímpico Internacional, 2020d, pp. 4-5)

Desde el decenio de 1990, el director general del COI, con sede en Lausana (Suiza), ha enumerado todos los directores generales, grupos de investigación e instituciones académicas olímpicas que han demostrado su compromiso permanente con la promoción de los estudios olímpicos.

Para facilitar el marco de funcionamiento de las OSC con el COI y respetar las propiedades olímpicas, como el símbolo, la bandera, el lema, los emblemas, la llama y las antorchas, definidas en la Carta Olímpica (Comité Olímpico Internacional 2020a), una directiva establece cuáles son los objetivos de este reconocimiento:

- Apoyar a los CEO universitarios proporcionando un marco claro para aquellos que contribuyen de forma continua a la enseñanza, creación o difusión de los conocimientos olímpicos.

- Subrayar la independencia de los CEO universitarios del COI y del Movimiento Olímpico.

- Regular el uso de la propiedad olímpica por los CEO universitarios y proteger la marca olímpica, de conformidad con lo dispuesto en la Carta Olímpica. (Comité Olímpico Internacional, 2020d, p. 3)

Los CEO reconocidos llevan a cabo actividades de investigación, educación o difusión de información relacionadas con el fenómeno olímpico. 
En abril de 2020, el CEO de Lausana había registrado oficialmente 43 CEO en 24 países (Comité Olímpico Internacional, 2020e). En 1989, la Universidad de Ontario Occidental, en Canadá, y la Universidad Autónoma de Barcelona, en España, inauguraron los primeros centros, el Centro de Estudios Olímpicos Internacionales (ICOS) y el Centro de Estudios Olímpicos y Deportivos de la ABU, respectivamente.

En la actualidad, 16 naciones tienen un solo centro y otras 8 tienen dos o más centros (tabla 4).

Tabla 4. Nombre de CEO por país.

\begin{tabular}{|l|c|c|}
\hline País & $\begin{array}{c}\text { Número de } \\
\text { naciones }\end{array}$ & Número de CEO \\
\hline $\begin{array}{l}\text { Argentina, Bélgica, Canadá, Chile, Francia, Israel, Italia, } \\
\text { Japón, México, Países Bajos, Nueva Zelanda, Noruega, } \\
\text { Rusia, Región del Océano Pacífico, Sudáfrica, Ucrania }\end{array}$ & 16 & 1 \\
\hline Alemania, Australia, Corea del Sur, Suiza & 4 & 2 \\
\hline Brasil, Estados Unidos & 2 & 3 \\
\hline China & 1 & 4 \\
\hline España & 1 & 9 \\
\hline
\end{tabular}

Fuente: elaboración propia.

Todos estos CEO están ubicados dentro de las universidades y generalmente se encuentran integrados en departamentos o institutos. Así, estas estructuras pueden llevar a cabo investigaciones universitarias y también otorgar diplomas: licenciaturas, maestrías y doctorados (tablas 5 y 6 ).

Tabla 5. Los nueve CEO en España en 2020.

\begin{tabular}{|l|c|c|}
\hline Nombre del CEO & Año de creación & Universidad española \\
\hline $\begin{array}{l}\text { The UAB Olympic and Sport Studies } \\
\text { Centre (CEO-UAB) }\end{array}$ & 1989 & Universitat Autònoma de Barcelona \\
\hline $\begin{array}{l}\text { Centro de Estudios Olímpicos } \\
\text { (CEO-UCAM) }\end{array}$ & 2001 & $\begin{array}{c}\text { Universidad Católica San Antonio de } \\
\text { Murcia, Facultad de Deporte }\end{array}$ \\
\hline Centro de Estudios Olímpicos & 2002 & Universidad de Santiago de Compostela \\
\hline Centro de Estudios Olímpicos & 2005 & $\begin{array}{c}\text { Universidad Autónoma de Madrid, } \\
\text { Facultad de Formación de Profesorado y } \\
\text { Educación }\end{array}$ \\
\hline
\end{tabular}




\begin{tabular}{|l|c|c|}
\hline Nombre del CEO & Año de creación & Universidad española \\
\hline Centro de Estudios Olímpicos & 2007 & Universidad de Vigo \\
\hline $\begin{array}{l}\text { Centre d'Estudis Olímpics J. A. } \\
\text { Samaranch }\end{array}$ & 2011 & Fundació Barcelona Olímpica \\
\hline Centro de Estudios Olímpicos & 2011 & $\begin{array}{c}\text { Universidad de Las Palmas de Gran } \\
\text { Canaria }\end{array}$ \\
\hline Centro de Estudios Olímpicos & 2011 & Universidad de Navarra \\
\hline $\begin{array}{l}\text { Càtedra d'Esport i Educació Fisica } \\
\text {-Centre d'Estudis Olímpics UdG }\end{array}$ & 2014 & Universitat de Girona \\
\hline
\end{tabular}

Fuente: elaboración propia.

Tabla 6. CEO en China, Estados Unidos y Brasil en 2020.

\begin{tabular}{|c|c|c|c|}
\hline Naciones & Nombre del CEO & $\begin{array}{l}\text { Año de } \\
\text { creación }\end{array}$ & Nombre de la universidad \\
\hline \multirow{4}{*}{ China } & Advanced Institute of Olympic Studies & 1994 & Beijing Sport University \\
\hline & Humanistic Olympic Studies Center & 2000 & Renmin University of China \\
\hline & Olympic Studies Centre & 2014 & Nantong University \\
\hline & $\begin{array}{l}\text { Beijing Institute for International } \\
\text { Olympic Studies }\end{array}$ & 2019 & $\begin{array}{l}\text { Capital University of } \\
\text { Physical Education and } \\
\text { Sports }\end{array}$ \\
\hline \multirow{3}{*}{ Estados Unidos } & Olympism and Development Center & 2010 & Brown University \\
\hline & $\begin{array}{l}\text { The Institute for Olympic Studies at the } \\
\text { H.J. Lutcher Stark Center for Physical } \\
\text { Culture and Sports }\end{array}$ & 2011 & $\begin{array}{l}\text { The University of Texas at } \\
\text { Austin }\end{array}$ \\
\hline & $\begin{array}{l}\text { Center for Sociocultural Sport and } \\
\text { Olympic Research }\end{array}$ & 2014 & California State University \\
\hline \multirow{3}{*}{ Brasil } & Olympic Studies Research Group & 2002 & $\begin{array}{l}\text { Pontifícia Universidade } \\
\text { Católica do Rio Grande } \\
\text { do Sul }\end{array}$ \\
\hline & Centre for Olympic Studies (ARETE) & 2006 & $\begin{array}{l}\text { Universidade Federal do } \\
\text { Espírito Santo }\end{array}$ \\
\hline & Olympic Research Group & 2014 & $\begin{array}{l}\text { Universidade do Estado do } \\
\text { Rio de Janeiro }\end{array}$ \\
\hline
\end{tabular}

Fuente: elaboración propia. 


\section{El Centro de Estudios e Investigaciones Olímpicas Universitarias (CEROU)}

EI CEROU es una federación de estudios e investigaciones de la Universidad de Franche-Comté (UFC). Su vocación es federar los estudios, la enseñanza y los trabajos de investigación relacionados con el olimpismo, realizados e impartidos dentro o fuera de la UFC. Para ello, coordina las colaboraciones entre los componentes y entidades de investigación de la UFC, con el fin de fomentar la aparición de estudios, enseñanza e investigación conjunta. Este Centro fue fundado por el UFC e involucra a todos sus componentes. Incluye a los miembros de oficio y a los miembros asociados que participan con derecho a voto en la Asamblea General del CEROU. EI CEROU se compromete a promover una política educativa y de investigación en los siguientes cuatro ejes estratégicos: investigación, enseñanza y formación, pericia, y promoción.

EI CEROU, que ha obtenido expresamente el apoyo de la CNOSF, del COl, del Ministerio de Deportes y del Comité Organizador de los Juegos Olímpicos de París 2024, es el primer CEO de Francia que tiene su sede en una universidad (Universidad de FrancheComté) y promueve la creación y la difusión de los conocimientos olímpicos.

Además de las cuatro condiciones mencionadas, para obtener el reconocimiento de CEO por parte del COI son esenciales dos criterios:

- Justificar las actividades pasadas y futuras de investigación, educación o difusión de información, relacionadas con el fenómeno olímpico en la universidad.

- Ser reconocido o tener una relación directa con el COI.

Estos dos criterios están ampliamente cubiertos por todos los proyectos de investigación académica realizados por la UFC, que desde 2009 cumple con una política comprometida con la promoción 
del deporte y los valores olímpicos, mediante diversos proyectos dirigidos a sus estudiantes, su personal y el público en general, en colaboración con numerosos agentes locales, nacionales e internacionales.

A continuación, se describen algunos ejemplos de eventos olímpicos organizados en la UFC.

Lugar y función del olimpismo en la educación en Europa (UFC, 2019)

Una jornada de estudio europea titulada Lugar y papel del olimpismo en la educación en Europa reunió en la UFC a académicos que dieron sus puntos de vista históricos, sociológicos o filosóficos sobre el fenómeno educativo vinculado al olimpismo. El objetivo de este día fue hacer una mirada crítica al olimpismo y al uso de sus valores con fines educativos. ¿En qué medida las experiencias pasadas, presentes y futuras pueden informarnos sobre la forma en que se utilizan, incluso se inventan o se apropian indebidamente, los valores olímpicos?, ¿son y pueden ser promovidos con fines educativos?

Por otra parte, los profesores de educación física y deportes, al menos en Francia, parecen haber tenido cierta reticencia desde los años setenta a utilizar referencias al olimpismo (República de los Deportes), a pesar de que son ampliamente compartidas en la escuela primaria a través de diversos experimentos (Revista EPS1, semanas deportivas de Besançon en 1984). ¿Puede el olimpismo, en un momento en que se está aplicando el Tratado de Lisboa, desempeñar un papel en la determinación de los valores comunes a Europa?

En última instancia, esta original e informativa jornada brindó la oportunidad de cuestionar la forma en que el mito olímpico opera en el campo de la educación, para comprender los pormenores de este fenómeno global. En particular, los oradores se preguntaron cómo se alimenta del peso de la imagen (campeones, historias 
antiguas renovadas), los rituales o las prácticas competitivas que transmite. No es importante entenderlo, incluso tampoco cuestionarlo, para admitir finalmente que todavía está muy vivo. Sin querer volver estrictamente a los antiguos griegos o a Coubertin, el olimpismo es un punto de referencia que debe tenerse en cuenta, aunque solo sea para posicionarlo en una sociedad que necesita puntos de referencia y que se inclina demasiado rápido a alabarlo o a condenarlo a los cielos.

\section{Día de la Cultura, el Deporte y la Educación}

En 2018, la UFC inaugura el primer Día de la Cultura, el Deporte y la Educación, estableciendo tres objetivos:

- Concienciar sobre los valores del olimpismo (excelencia, solidaridad, respeto).

- Cambiar la forma de ver la discapacidad descubriendo los deportes paralímpicos.

- Facilitar la transición de la escuela secundaria a la universidad.

Durante este día, los estudiantes participan en talleres agrupados en torno a los siguientes tres temas:

- Cultura, a través de exposiciones (carteles olímpicos, objetos, etc.) y conferencias.

- Deporte, con eventos físicos basados en la ayuda mutua, la solidaridad y la ciudadanía en relación con los temas de la discapacidad, el envejecimiento o, de forma general, la integración en la sociedad.

- Educación, con la presentación oral hecha por los estudiantes acerca de su trabajo, testimonios de atletas, un taller de ciudadanía, etc. 
Así las cosas, tres pasos esenciales han permitido la creación del primer CEROU francés:

- El 20 de septiembre de 2019, durante el Día Internacional del Deporte Universitario (Unesco, 2019), el Comité Regional Olímpico y Deportivo de la región de Borgoña-Franco Condado firmó un acuerdo con la UFC para convertirse en socio oficial del CEROU. Esta fecha es muy simbólica, ya que todos los Estados miembros de la Unesco, durante la 38. ${ }^{a}$ reunión de la Conferencia General en París en noviembre de 2015, adoptaron este día que:

Tiene por objeto garantizar y preservar las actividades y programas deportivos en las instituciones de enseñanza superior para estudiantes y ciudadanos, a fin de fomentar la ética, los sistemas de valores, la lucha contra el dopaje, el juego limpio, la educación física, los estilos de vida saludables, la calidad de vida, la educación física de calidad, la igualdad entre los géneros y la inclusión social, y garantizar así una educación física de alta calidad. (Unesco, 2019)

Esta ceremonia, que se llevó a cabo bajo el alto patrocinio del Ministro de Deportes y del presidente del COI, permitió lanzar el proyecto CEROU en presencia del Ministerio de Deportes, del COI y del Comité Internacional de los Juegos de la Francofonía, París 2024.

- El 10 de diciembre de 2019, el Consejo de Administración de la UFC votó por unanimidad la creación oficial del primer CEROU francés.

- El 12 de marzo de 2020, el Consejo de Administración de la Universidad de Franche-Comté hizo el nombramiento del director del CEROU.

Los valores del olimpismo (excelencia, amistad, respeto), del paralelismo (coraje, determinación, igualdad, inspiración) y, más 
generalmente, del deporte son educativos y universales. Estos valores pueden ser fácilmente transmitidos desde una edad muy temprana. Encuentran un verdadero campo de expresión en la enseñanza en la escuela y la universidad. Con este espíritu, el Ministerio de Educación Nacional de Francia, en apoyo a la candidatura de París 2024, declaró oficialmente el año escolar 2016-2017 como el Año del Olimpismo de la Escuela a la Universidad, con el objetivo de "vincular la práctica del deporte a una ambición educativa, cultural o cívica basada en el olimpismo y sus valores" (Ministerio de Educación Nacional, Enseñanza Superior e Investigación, 2016).

De acuerdo con la anterior declaración, el CEROU de la UFC persigue dos objetivos principales.

- Facilitar y acelerar la labor de investigación mediante el establecimiento de diversos aspectos: una red de investigadores; simposios y seminarios internacionales; intercambio y acogida de investigadores y estudiantes; transferencia de conocimientos académicos a través de los demás centros de estudios olímpicos; desarrollo de recursos para investigadores, profesores y estudiantes visitantes, con el fin de promover los conocimientos y estudios olímpicos; tutoría de estudiantes de maestría y doctorado; publicaciones y comunicaciones de los estudios realizados y dirigidos en el Centro.

En síntesis, con este primer objetivo se busca:

- Fomentar el trabajo en asociación interdisciplinaria.

- Fortalecer los vínculos y sinergias científicas entre los laboratorios de investigación del CEROU.

- Fortalecer la estructuración de las entidades existentes o futuras para promover la creación de redes.

- Promover la visibilidad de los laboratorios de investigación, abriéndolos al mercado internacional o impulsando sus conocimientos técnicos. 
- Fomentar los intercambios entre los laboratorios de investigación del CEROU y el mundo socioeconómico.

- Con la creación del CEROU, la UFC se distingue de las demás universidades y aumenta su visibilidad en Francia y en el extranjero. Este reconocimiento internacional muestra una fuerte ambición por innovar y destacar entre las demás universidades francesas. EI CEROU se ajusta a las directivas ministeriales para promover la educación mediante el deporte y para poner los valores cívicos y deportivos en el centro de la enseñanza a través del olimpismo.

Para la UFC, el CEROU es una verdadera oportunidad que le permite proyectar:

- Su visibilidad y reconocimiento en Francia y el extranjero.

- Su ambición de innovar y sobresalir.

- Su investigación sobre el olimpismo y las paralimpiadas (investigación, publicaciones).

- Sus acciones y conocimientos para los investigadores y estudiantes.

- Asimismo, le permite aumentar su actual apoyo nacional e internacional, como el COI, CNOSF, París 2024 y los que vengan en un futuro.

\section{Conclusiones}

Desde la elección de París (ciudad anfitriona de la Olimpiada de la Era Moderna XXXIII), los ministerios de Educación Nacional, Educación Superior, Investigación e Innovación y Deportes han multi- 
plicado las acciones y los llamamientos a la participación a través de diversos eventos, como la Semana Olímpica y Paralímpica y el Día Olímpico. Con el mismo espíritu de estos eventos, en 2018 se creó la etiqueta Generación 2024 para escuelas y universidades.

El olimpismo, verdadero vector educativo, se considera una oportunidad para ayudar a transmitir y enseñar ciertos valores en la escuela, como la cooperación o la ayuda mutua. Los tres valores fundamentales del olimpismo (excelencia, respeto y amistad), desarrollados en torno a cinco temas educativos (aprender la alegría del esfuerzo; aprender el juego limpio; aprender el respeto por los demás; aprender a buscar la excelencia y aprender el equilibrio entre el cuerpo, la voluntad y la mente), pueden ser parte integrante de los objetivos establecidos en los programas escolares y universitarios (Ministère de I'Education Nationale de la Jeunesse et des Sports, 2018).

Por lo tanto, los objetivos de la educación para formar un ciudadano responsable están muy cerca de los principios establecidos en la Carta Olímpica: "La finalidad del olimpismo es poner el deporte al servicio del desarrollo armonioso de la humanidad con miras a promover una sociedad pacífica preocupada por la preservación de la dignidad humana" (Comité Olímpico Internacional, 2020a, p. 9).

El olimpismo sigue siendo un componente ineludible de nuestra sociedad al que los estudiantes aluden en diversas ocasiones, por ejemplo los Juegos Olímpicos. El profesor, en búsqueda de elementos para sus lecciones, puede referirse al modelo olímpico, con el fin de enseñar educación moral y cívica, geopolítica, interculturalidad, etc. La libertad pedagógica les permite a los profesores integrar sus propias representaciones del olimpismo excluyendo todas sus contradicciones.

La creación del CEROU, una nueva herramienta universitaria única en Francia y que se inscribe en la línea de las investigaciones actuales sobre la educación olímpica (Academia Olímpica Internacional, 
2020), permite aprehender, analizar, comprender y describir los comportamientos de los actores sobre el conjunto de las acciones/ eventos olímpicos desarrollados en el seno o fuera de la escuela y la universidad.

A pesar de las herramientas y programas desplegados, surge una incógnita: ¿después de los Juegos de París 2024, continuará esta dinámica puesta en marcha por la ciudad anfitriona en las escuelas y universidades, y estará allí el legado olímpico previsto desde la candidatura?

\section{Referencias}

Academia Olímpica Internacional. (2020). Movimiento y gobierno olímpico: el papel de la educación olímpica. IOA Journal 11\&12. http:// ioa.org.gr/wp-content/uploads/2018/01/j-11-12_en-fr-1.pdf

Carta de los Ministros de Educación Superior, Investigación e Innovación y Deportes. (2018). A los Presidentes de las Universidades. París.

Comité Olímpico Internacional. (1978). Carta Olímpica, Comité Olímpico Internacional, Lausana. https://stillmed.olympics.com/media/ Document\%20Library/OlympicOrg/General/FR-Olympic-Charter.pdf

Comité Olímpico Internacional. (2006). Foco: Deporte, Educación y Cultura. Revista Olímpica. https://www.olympic.org/fr-FR/Home/ Documents/OLD_Documents/Olympic\%20Review\%20Focus/FOCUS\%20 SPORT\%20EDUCATION\%20AND\%20CULTURE

Comité Olímpico Internacional. (2007). Enseñar valores: un manual de educación olímpica. file:///F:/Documents/Articles\%20revues/Education\%20 olympique/kit\%20PEVO\%202008/PEVO\%20Enseigner\%20les\%20 valeurs\%20manuel\%20d'éducation $\% 20$ olympique\%20en\%20Français.pdf

Comité Olímpico Internacional. (2008). Focus on PEVO. https://stillmed. olympic.org/media/Document\%20Library/OlympicOrg/Documents/ Olympic-Review/Focus/2000-2009/Focus-Sur-PEVO-Le-programme-deducation-aux-valeurs-olympiques-Septembre-2008.pdf 
Comité Olímpico Internacional. (2014). Agenda Olímpica 2020. https:// stillmed.olympic.org/media/Document\%20Library/OlympicOrg/ Documents/Olympic-Agenda-2020/Agenda-olym pique-2020-20-20Recommendations.pdf

Comité Olímpico Internacional. (2017). Hoja de información. Día Olímpico (23 de junio), Comité Olímpico Internacional. Lausana.

Comité Olímpico Internacional. (2020a). Carta Olímpica. Comité Olímpico Internacional, Lausana.

Comité Olímpico Internacional. (2020b). Programa de Educación en Valores Olímpicos. https://www.olympic.org/fr/programme-valeurs-eteducation-olympique

Comité Olímpico Internacional. (2020c). Sobre la OVEP. https://www. olympic.org/fr/programme-valeurs-et-education-olympique/a-proposdu-pevo

Comité Olímpico Internacional. (2020d). Directrices para los centros universitarios de estudios e investigación olímpicos, Centro de Estudios Olímpicos. Lausana.

Comité Olímpico Internacional. (2020e). Centres d'études olympiques dans le monde. https://stillmedab.olympic.org/media/Document\%20 Library/OlympicOrg/Olympic-Studies-Centre/Academic-Activities-andNetwork/Olympic-Studies-monitoring/Centres-universitaires-d-etudeset-de-recherches-olympiques-dans-le-monde.pdf

Consejo de Ministros. (2017). El Programa de Legado Estatal para los Juegos Olímpicos y Paralímpicos de 2024. https://www.gouvernement. $\mathrm{fr} /$ conseil-des-ministres/2017-03-22/le-programme-heritage-de-l-etatpour-les-jeux-olympiques-et-

Frécon, É. (2016). ¿Cuál es el balance del Año del Deporte de la escuela a la universidad? Revista EP\&S, 372(9-10), 38-39. http://circulaires.ia80. ac-amiens.fr/2018-2019/04_03_2019_generation_2024_(cahier_des_ charges)_classes_olympiques.pdf 
Georgiadis, K. (2007) "Définition de l'éducation olympique", en Le sport et un monde en harmonie : le rôle de la culture et de l'éducation olympique, 5e Forum mondial sur le sport, l'éducation et la culture, 22-24 octobre 2006 Pékin, Comité International Olympique, Lausanne, p. 217.

Ministerio de Educación Nacional. (2018). Apéndice - Programa de Educación Moral y Cívica en Escuelas y Colegios (Ciclos 2, 3 y 4). Boletín oficial, 30.

Ministerio de Educación Nacional. (2016). Día Nacional de los Deportes Escolares (JNSS) - Miércoles 14 de septiembre de 2016. http://www. education.gouv.fr/pid285/bulletin_officiel.html?cid_bo=102494

Ministerio de Educación Nacional y Juventud. (2016). Le parcours citoyen del'élève.https://www.education.gouv.fr/pid285/bulletin_officiel.html? cid_bo=103533

Ministerio de Educación Nacional y Juventud. (2018). L'école promotrice de santé. https://cache.media.eduscol.education.fr/file/Sante/84/2/VM_ EcolePromotriceSante_1240842.pdf

Ministerio de Educación Nacional y Juventud, Ministerio de Agricultura y Alimentación y Ministerio de Deportes. (2018). Desarrollar puentes entre el mundo escolar y el movimiento deportivo para fomentar la práctica física y deportiva de los niños y jóvenes. Convocatoria de solicitudes y especificaciones Generación 2024.

Ministerio de Educación Nacional, Enseñanza Superior e Investigación. (2015). Año del Deporte desde la Escuela a la Universidad 2015-2016. http://cache.media.education.gouv.fr/file/ASEU/24/4/ASEU_Plaquette_ presentation_septembre2015_471244.pdf

Ministerio de Educación Nacional, Enseñanza Superior e Investigación. (2016). Año del Olimpismo, de la escuela a la universidad. Circular n.o 2016-126. http://www.education.gouv.fr/pid285/bulletin_officiel. html?cid_bo $=105548$ 
Ministerio de Educación Nacional, Juventud y Deportes. (2020a). 8.o Día Nacional del Deporte Escolar: iEl espíritu del Olimpismo es contagioso! https://www.education.gouv.fr/8e-journee-nationale-du-sportscolaire-I-esprit-de-I-olympisme-est-contagieux-4784

Ministerio de Educación Nacional, Juventud y Deportes. (2020b). Día Nacional de los Deportes Escolares. https://eduscol.education.fr/cid57 378/journee-nationale-du-sport-scolaire.html

Ministerio de Educación Nacional, Juventud y Deportes. (2020c). Día Olímpico el 23 de junio de 2020. https://eduscol.education.fr/cid152593/ journee-olympique-du-23-juin-2020.html

Ministerio de Educación Superior, Investigación e Innovación. (2019). Día Olímpico de 2019: 24 nuevos establecimientos premiados con la etiqueta Generación 2024. https://www.enseignementsup-recherche.gouv.fr/ cid143156/journee-olympique-2019-24-nouveaux-etablissementslaureats-du-label-generation-2024.html

Ministerio de Educación Superior, Investigación e Innovación. (2020). Label Génération 2024: 10 nuevos establecimientos premiados. https://www.enseignementsup-recherche.gouv.fr/cid152646/www. enseignementsup-recherche.gouv.fr/cid152646/label-generation-202410-nouveaux-etablissements-laureats.html

Ministerio de Educación Superior, Investigación e Innovación y Ministerio de Deportes. (2018). Etiquetando "Generación 2024". http:// cache.media.education.gouv.fr/file/janvier2018/35/3/Labellisation_ Generation_2024_et_Cahier_des_charges_880353.pdf

Monnin, É. (2019). Desde el "Año del Olimpismo, de la escuela a la etiqueta de la universidad" hasta "la Generación 2024". Etiqueta olímpica: Un ejemplo dentro de la Universidad de Franche-Comté. Diagoras. Revista Académica Internacional de Estudios Olímpicos. 3.

Navarranne, O. (2019, 2 de septiembre). LA JNSS PUISSANCE 10. Sportmag. fr. https://www.sportmag.fr/sport-scolaire/la-jnss-puissance-10/ 
París 2024. (2020a). 30' D'activité Physique Quotidienne. https:// generation.paris2024.org/30-dactivite-physique-quotidienne

París 2024. (2020b). Semaine olympique et paralympique. 5ème édition. https://medias-generation.paris2024.org/2020-11/Guide\%20 Semaine\%200lympique\%20et\%20Paralympique\%202021.pdf

París, ciudad candidata a los Juegos Olímpicos de 2024. (2016). Expediente de candidatura. Fase 1.

París, ciudad candidata a los Juegos Olímpicos de 2024 y Ministerio de la Ciudad, la Juventud y los Deportes. (2017). El deporte al servicio de la sociedad. https://www.sports.gouv.fr/IMG/pdf/paris2024_ programmeheritage.pdf

Plateau, B. (2018). Procedimiento para solicitar el sello "Generación 2024" para las instituciones de enseñanza superior. París.

Remmelkoor, R. (2007). Aplicación de la educación olímpica por las Academias Olímpicas Nacionales. 5. - Foro Mundial sobre el Deporte, la Educación y la Cultura, 22-24 de octubre de 2006 Beijing. Comité Olímpico Internacional. Lausana.

Revue EPS. (2017). 98 projets labellisés, $n^{\circ} 374$, p. 5.

Unesco. (2019). Journée internationale du sport universitaire. https:// fr.unesco.org/events/journee-internationale-du-sport-universitaire

Universidad de Franche-Comté. (2018a). L'université de Franche-Comté Labellisée "Génération 2024». http://actu.univ-fcomte.fr/article/luniver site-de-franche-comte-labellisee-generation-2024-007068 